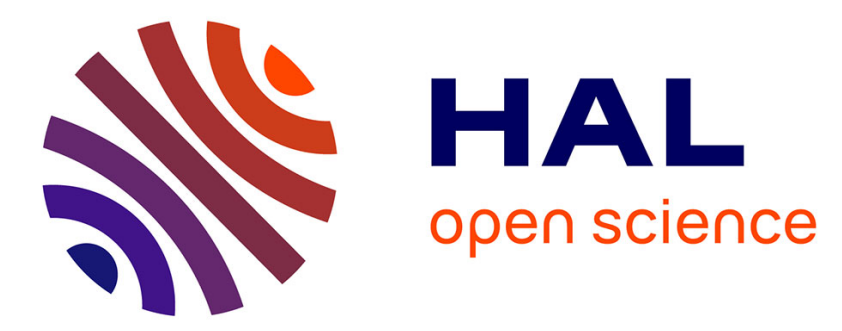

\title{
How our beliefs contribute to interpret actions
}

Guillaume Aucher

\section{To cite this version:}

Guillaume Aucher. How our beliefs contribute to interpret actions. CEEMAS - Central and Eastern European Conference on Multi-Agent Systems - 2005, Sep 2005, Budapest, Hungary. inria00556095v2

\section{HAL Id: inria-00556095 \\ https://inria.hal.science/inria-00556095v2}

Submitted on 8 Sep 2013

HAL is a multi-disciplinary open access archive for the deposit and dissemination of scientific research documents, whether they are published or not. The documents may come from teaching and research institutions in France or abroad, or from public or private research centers.
L'archive ouverte pluridisciplinaire HAL, est destinée au dépôt et à la diffusion de documents scientifiques de niveau recherche, publiés ou non, émanant des établissements d'enseignement et de recherche français ou étrangers, des laboratoires publics ou privés. 


\title{
How Our Beliefs Contribute to Interpret Actions $^{\star}$
}

\author{
Guillaume Aucher ${ }^{\star \star}$ \\ University of Otago (NZ) - University Paul Sabatier (F) \\ aucher@cs.otago.ac.nz
}

\begin{abstract}
In update logic the interpretation of an action is often assumed to be independent from the agents' beliefs about the situation (see BMS04] or Auc05]). In this paper we deal with this type of phenomenon. We also deal with actions that change facts of the situation. We use probability to model the notion of belief and our probabilistic update mechanism satisfies the AGM postulates of belief revision.
\end{abstract}

Often in everyday life we interpret an action on the basis of our beliefs about the situation. For example, assume that you see somebody drawing a ball from an urn containing black balls and white balls. If you believe there is no particular distribution in the urn then you will consider it equally probable that he draws a white ball or a black ball; but if you believe there are more black than white balls in the urn then you will consider it more probable than he draws a black ball than a white ball: the interpretation of the same action is different in both cases. The literature about update logic does not deal with this kind of phenomenon (see BMS04 or Auc05). Likewise, actions that change the truth of propositions are also neglected in the literature about update logic although they are quite common in everyday life and hence interesting to formalize.

This paper is a continuation of Auc05] and solves the problems raised there. However, instead of using plausibilities we use probabilities because in this context it is technically easier to deal with probability, and because I believe the modeling of the notion of belief is better rendered with a subjective probability. A novelty is also our use of infinitesimals in order to express what would surprise the agents and by how much.

In Sect.1 we motivate and define a structure slightly different from the hyperreals where the probabilities of worlds take value. In Sect.2 we present the core of the system in three parts : static (where we model static situations), dynamic (where we model actions) and the update mechanism. Finally, in Sect.3 we compare it with the AGM postulates and other relevant literature.

\footnotetext{
* An extended version of this paper with proofs is available on my homepage.

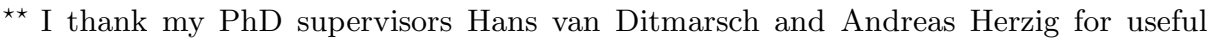
comments and discussions.
} 


\section{Mathematical Preliminaries}

In the proposed system, the probabilities of worlds and formulas will take value in a particular mathematical structure $(\mathbb{V}, \lesssim$ ) (abusively denoted $(\mathbb{V}, \leq)$ ) different from the real numbers, based on hyperreal numbers $\left({ }^{*} \mathbb{R}, \leq\right.$ ) (see Adams75. which uses them as well to give a probabilistic semantics to conditional logic).

Roughly speaking, hyperreal numbers are an extension of the real numbers to include certain classes of infinite and infinitesimal numbers. A hyperreal number, typically denoted $\varepsilon$, is said to be infinitesimal iff $|\varepsilon|<1 / n$ for all integers $n$; and finite if $|x|<n$ for some integer $n$ (in that case $S t(x)$ is the unique real number closest to $x$ ). For an account on the hyperreal numbers, see chapter 1 of Keis86.

What we would like to do is to approximate our expressions. That is to say, in case an hyperreal number $a$ is infinitely smaller than $b$ (that is to say, there is an infinitesimal $\varepsilon$ such that $a=\varepsilon . b$ ), then we would want $b+a=b$; for example ' $1+\varepsilon=1$ ', ${ }^{\prime} \varepsilon+\varepsilon^{2}=\varepsilon^{\prime}, \ldots$ Unfortunately, the hyperreal numbers do not allow us to do that, so we are obliged to introduce a new structure $(\mathbb{V}, \lesssim \sqrt{1}$ defined in the footnote 1 .

\section{Dynamic Proba-Doxastic Logic}

\subsection{The Static Part}

Definition 1. A proba-doxastic model (pd-model) $M=\left(W,\left\{\sim_{j} ; j \in G\right\},\left\{P_{j} ; j\right.\right.$ $\left.\in G\}, V, w_{0}\right)$ is a tuple where:

1. $W$ is a finite set of possible worlds.

2. $w_{0}$ is the possible world corresponding to the actual world.

3. $\sim_{j}$ is an equivalence relation defined on $W$ for each agent $j$.

4. $P_{j}$ is an operator for each agent $j$ which assigns to each world $w$ a number in ]0;1] such that $\sum\left\{P_{j}(v) ; v \sim_{j} w\right\}=1\left(^{*}\right)$.

5. $V$ is a valuation.

6. $G$ is a set of agents.

Intuitive Interpretation. Items 1,2,5,6 are clear. It remains to give interpretations for items 3 and 4 . The probabilistic operator $P_{j}$ together with $\sim_{j}$ intuitively model the epistemic state of mind of any agent $j \in G$. We set $w \sim_{j} v$ iff agent $j$ can not distinguish world $w$ from world $v$. This relation does not model the notion of knowledge and we do not deal with this notion in this paper (see general conclusion).

Among the worlds that agent $j$ can not distinguish, there are some that $j$ conceives as potential candidates for the world in which $j$ dwells, and some

${ }^{1} \mathbb{V}$ is the quotient structure of the set of positive hyperreal numbers ${ }^{*} \mathbb{R}^{+}$by the equivalence relation $\approx$ defined by $x \approx y$ iff $\left(\left(\operatorname{St}\left(\frac{x}{y}\right)=1\right.\right.$ and $\left.y \neq 0\right)$ or $\left.x=y=0\right)$. We define a total order $\lesssim$ on $\mathbb{V}$ by $\bar{x} \lesssim \bar{y}$ iff there are $x \in \bar{x}, y \in \bar{y}$ such that $x \leq y$. Elements of $\mathbb{V}$ containing an infinitesimal (resp. real) are abusively called infinitesimals (resp. reals) and we abusively denote $\lesssim$ by $\leq$. 
that $j$ would be surprised to hear somebody claiming that they correspond to the world in which $j$ dwells (whatever it is). The first ones are called conceived worlds and the second surprising worlds. The conceived worlds are assigned by $P_{j}$ a real value and the surprising worlds are assigned an infinitesimal value (both different from 0). For example, some people would be surprised to hear somebody claiming that some swans are black, although it is true. So for them the actual world is a surprising world, indistinguishable from the only conceived world where all swans are white.

Of course $j$ might conceive some (conceived) worlds as better candidates than others and this is expressed by the value of the probability of the world: the larger the real probability value of the (conceived) world is, the more likely it is for $j$. But that is the same for the surprising worlds: $j$ might be more surprised to hear some worlds than others. For example, if you play poker with your brother (or a friend), you will never suspect that he cheats. However he does so, and so carefully that you do not suspect anything. Then at the end of the game if he announces to you that he has cheated, you will be surprised (although it is something true in the actual world, which is then a surprising world). But you will be even more surprised if he tells you that he has cheated five times than if he has cheated only once. So the world where he has cheated five times will be more surprising than the world where he has cheated once, and these are both surprising worlds for you. Infinitesimals enable us to express this: the larger the infinitesimal probability value of the (surprising) world is, the less $j$ would be surprised by this world. Anyway, that is why we need to introduce these hyperreal numbers: to express these degrees of surprise that can not be expressed by a single number like 0 (which then becomes useless for us).

Finally, the natural condition $(*)$ ensures us that $\left([w]_{j}, \mathcal{P}\left([w]_{j}\right), P_{j}\right)$ (with $\left.\left.[w]_{j}:=\left\{v ; v \sim_{j} w\right\}\right)\right)$ is a (nonstandard) probability space.

So, dwelling in one of the world of $[w]_{j}, j$ does not think consciously that her respective surprising worlds in $[w]_{j}$ are possible (unlike conceived worlds), she is just not aware of them. So they are useless to represent her beliefs which I assume are essentially conscious. But still, these worlds are relevant for the (objective) modelisation. Indeed they provide some information about the epistemic state of mind of $j$ : namely what would surprise her. Intuitively, something that you do not consider consciously as possible and that contradicts your beliefs is often surprising for you when it is claimed by somebody else. These worlds will moreover turn out to be very useful technically in case $j$ has to revise her beliefs.

Definition 2. The syntax of the language $\mathcal{L}_{S t}$ is defined by,

$\phi:=\perp|p| \neg \phi|\phi \wedge \psi| P_{j}(\phi) \geq x \mid C_{j} \phi$ where $x \in[0 ; 1[$.

Its semantics is defined by,

$M, w \models P_{j}(\phi) \geq x$ iff $\sum\left\{P_{j}(v) ; w \sim_{j} v\right.$ and $\left.M, v \models \phi\right\} \geq x$

$M, w \models C_{j} \phi$ iff $\sum\left\{P_{j}(v) ; w \sim_{j} v\right.$ and $\left.M, v \models \phi\right\}=1$.

$M, w \models P_{j}(\phi) \geq x$ should be read "in world $w$ and for $j, \phi$ has a probability greater than $x " . M, w \models C_{j} \phi$ should be read "in world $w, j$ is convinced (sure) of $\phi "$. 
Note that above, if $x$ is real then only the conceived worlds have to be considered in the sum (see Sect.1). Similarly the semantics of $C_{j}$ amounts to say that $\phi$ is true only in all the conceived worlds of $[w]_{j}$ (see Sect.1). This is not surprising since only the conceived worlds describe the beliefs of the agent as we just said above. So it is quite possible to have a surprising actual world where $\neg \phi$ is true and still $j$ being convinced of $\phi$ (i.e. $C_{j} \phi$ ): see the swan example above with $\phi:=$ "All swans are white".

Moreover, we can also express in this language what would surprise the agent, and by how much. Indeed, in case $x$ is infinitesimal, $M, w \models\left(P_{j}(\phi)=x\right)$ should be read "in world $w, j$ would be surprised with intensity $x$ to hear somebody claiming that $\phi "$. (Note that the smaller $x$ is, the higher the intensity of surprise is.)

Remark 1. If we define the operator $B_{j} \phi$ by $P_{j}(\phi)>\frac{1}{2}$, then the meaning of the operators $B_{j}$ and $C_{j}$ are respectively exactly the same as the Lenzen's notion of (weak) belief and conviction (see Len03]). Often these notions are confused and we use in everyday life the same word "belief" to denote both of them.

Example 1. Consider two friends A and B in a fair, and an urn containing $n=$ $2 . k>0$ balls which are either white or black. A knows how many black balls there are in the urn but B does not know it. Now say there are actually 0 black ball in the urn. This situation is depicted in Fig.1, where $p_{i}$ stands for: "there are $i$ black balls in the urn " and the double bordered world is the actual world. In Fig. 1 the probabilities of worlds are $P_{A}(x)=1$ and $P_{B}(x)=\frac{1}{n+1}$ for all worlds $x$, so $\mathrm{B}$ believes there is no particular distribution in the urn.

Example 2. Consider the same example as before but now B is convinced (sure) that there are more black balls than white balls (i.e. $C_{B}\left(p_{k+1} \vee \ldots \vee p_{n}\right)$ ). For example her friend A might have lied to her by telling her so, and she fully believed him. This situation is still depicted in Fig.1 but the probabilities of worlds here are $P_{A}(x)=1$ for all worlds $x$, and $P_{B}\left(w_{i}\right)=\epsilon$ for all $i \in\{0, . ., k\}$, $P_{B}\left(w_{i}\right)=\frac{1}{k}$ for all $i \in\{k+1, . ., n\}$. The worlds where there are less black balls than white balls are all (equally) surprising.

$$
\underline{w_{0}: p_{0}}-\underline{B}-w_{k}: p_{k} \quad{ }^{B} w_{k+1}: p_{k+1}-w_{n}: p_{n}
$$

Fig. 1. 'urn' Examples 1 and 2 (without specifications of the probabilities of worlds for each example: see text)

Throughout this paper we apply our system to the example of the introduction. So note that Examples 1 and 2 only differ in what the agent B believes.

\subsection{The Dynamic Part}

Definition 3. A generic action model is a structure $\boldsymbol{\Sigma}=\left(\Sigma, S,\left\{\sim_{j} ; j \in G\right\}\right.$, $\left\{P_{j}^{\Gamma} ; \Gamma\right.$ is a maximal consistent subset of $S$ and $\left.j \in G\right\},\left\{\right.$ Post $\left.\left._{\sigma} ; \sigma \in \Sigma\right\}, \sigma_{0}\right)$ where 
1. $\Sigma$ is a finite set of possible actions.

2. $\sigma_{0}$ is the actual action.

3. $\sim_{j}$ is an equivalence relation defined on $\Sigma$ for each agent $j$.

4. $S$ is a set of formulas of $\mathcal{L}_{S t}$ and their negations.

5. $P_{j}^{\Gamma}$ is an operator indexed by each agent $j$ and each maximal consistent subset $\Gamma$ of $S$ which assigns to each possible action a number in [0;1], such that for each possible action $\sigma$ and agent $j_{0}$ if $P_{j_{0}}^{\Gamma}(\sigma)=0$ then $P_{j}^{\Gamma}(\sigma)=0$ for all $j \in G\left({ }^{*}\right)$.

6. Post $_{\sigma}$ is a function which takes as argument a propositional letter $p$ and gives two formulas of $\mathcal{L}_{S t}$ Post $_{\sigma}(p):=\left(\right.$ Post $_{\sigma}^{+}(p)$, Post $\left._{\sigma}^{-}(p)\right)$ such that $\models$ $\neg\left(\right.$ Post $_{\sigma}^{+}(p) \wedge$ Post $\left._{\sigma}^{-}(p)\right)(* *)$.

7. $G$ is a set of agents.

Intuitive Interpretation. Items $1,2,3,7$ are totally similar to definition 1 . It remains to give interpretation to items $4,5,6$ of the definition.

Item 4. $S$ corresponds to the set of formulas (and their negation) that are relevant for the agents in a particular world in order to assign a probability to an action occurring in this world. Consequently, the larger the size of $S$ will be, the more the interpretation by the agents of the actions will be dependant on their beliefs (see Sect.2.3).

Item 5. $P_{j}^{\Gamma}$ is the probability for $j$, if she assumes she is in a particular world where the set of formulas $\Gamma$ is true, that the action $\sigma$ is occurring.

Just as in the static case, relatively to a world $w$ satisfying $\Gamma$ and among indistinguishable actions for the agent $j$, we have conceived actions (which are assigned a real number) and surprising actions (which are assigned an infinitesimal). The former are possible actions that the agent conceives as possible candidates while one of the indistinguishable actions actually takes place. The latter are possible actions that $j$ would be surprised to hear somebody claiming that they took place while one of the indistinguishable actions actually took place. For example, if you play poker with your brother (or a friend) and at a certain point he cheats while you do not suspect anything, then the actual action of cheating will be a surprising action for you (of value $\varepsilon$ ) and will be indistinguishable for you from the conceived action where nothing particular happens (of value 1 ).

Just as in the static case, the relative strength for $j$ of the indistinguishable actions (conceived and surprising), relatively to a world where $\Gamma$ is true, is expressed by the value of the operator $P_{j}^{\Gamma}$.

Finally, note that we can have $P_{j}^{\Gamma}(\sigma)=0$. This means that the action $\sigma$ can not physically be performed in a world where $\Gamma$ is true. This impossibility is public and inherent to the possible action, that is why we have the condition $(*)$. That replaces the notion of precondition in BMS04 and Auc05.

From now on, we note $\boldsymbol{P}_{j}^{\boldsymbol{w}}(\sigma):=\boldsymbol{P}_{j}^{\Gamma}(\sigma)$ for the unique $\boldsymbol{\Gamma}$ such that $M, \boldsymbol{w} \models \Gamma$.

Item 6. The function Post $_{\sigma}$ deals with the problem of determining what facts will be true in a world after the action $\sigma$ takes place. Intuitively, $\operatorname{Post}_{\sigma}^{+}(p)$ (resp. 


$$
\begin{gathered}
S=\left\{p_{i}, \neg p_{i} ; i=0 . . n\right\} . \\
P_{B}^{\left\{p_{i}\right\}}(\sigma)=\frac{i}{n}, P_{B}^{\left\{p_{i}\right\}}(\tau)=1-\frac{i}{n} \text { for all } i . \\
P_{A}^{\left\{p_{i}\right\}}(\sigma)=1 \text { for all } i \neq 0, P_{A}^{\left\{p_{0}\right\}}(\sigma)=0 . \\
P_{A}^{\left\{p_{i}\right\}}(\tau)=1 \text { for all } i \neq n, P_{A}^{\left\{p_{n}\right\}}(\tau)=0 . \\
P_{A}^{\Gamma}(\sigma)=P_{A}^{\Gamma}(\tau)=P_{B}^{\Gamma}(\sigma)=P_{B}^{\Gamma}(\tau)=0 \text { for all } \Gamma \neq\left\{p_{i}\right\} . \\
\operatorname{Post}_{\sigma}^{+}\left(p_{n}\right)=\perp, \operatorname{Post}_{\sigma}^{-}\left(p_{n}\right)=\top . \\
\operatorname{Post}_{\sigma}^{+}\left(p_{i}\right)=p_{i+1}, \operatorname{Post}_{\sigma}^{-}\left(p_{i}\right)=\neg p_{i+1} \text { for all } i<n . \\
\operatorname{Post}_{\tau}^{+}\left(p_{n}\right)=\perp, \operatorname{Post}_{\tau}^{-}\left(p_{n}\right)=\top . \\
\operatorname{Post}_{\tau}^{+}\left(p_{i}\right)=p_{i}, \operatorname{Post}_{\tau}^{-}\left(p_{i}\right)=\neg p_{i} \text { for all } i<n .
\end{gathered}
$$

Fig. 2. A draws a (white) ball, observes it and puts it in his pocket

Post $\left._{\sigma}^{-}(p)\right)$ represents the precondition in any world $w$ for $p$ to become true (resp. false) in $(w, \sigma)$ after the performance of $\sigma$. The role of condition (**) is to avoid the case where the performance of an action $\sigma$ in a world $w$ could provoke at the same time both $p$ and $\neg p$ to be true in the resulting world $(w, \sigma)$.

Example 3. Now, let us resume the 'urn' example. Consider the action whereby A draws a ball from the urn (which is actually white), looks at it and puts it in his pocket, B sees A doing that but can not see the ball. This action is depicted in Fig.2. The maximal consistent sets are represented by their 'positive' components, so $\left\{p_{i}\right\}$ refers to the set $\left\{p_{i}, \neg p_{k} ; k \neq i\right\}$.

It looks quite complicated but the ideas behind are quite simple. Action $\sigma$ (resp. $\tau$ ) stands for "A draws a black (resp. white) ball, observes it and puts it in his pocket". Clearly B can not distinguish $\sigma$ from $\tau$. However if B assumes she is in a world where there are $i$ black balls then for her the probability that A draws a black (resp. white) ball is $\frac{i}{n}$ (resp. $\left.1-\frac{i}{n}\right): P_{B}^{\left\{p_{i}\right\}}(\sigma)=\frac{i}{n}$ and $P_{B}^{\left\{p_{i}\right\}}(\tau)=1-\frac{i}{n}$. Moreover there can not be $n$ black balls in the urn since A put one ball in his pocket $\left(\operatorname{Post}_{\sigma}^{+}\left(p_{n}\right)=\perp, \operatorname{Post}_{\sigma}^{-}\left(p_{n}\right)=\top\right.$ and $\left.\operatorname{Post}_{\tau}^{+}\left(p_{n}\right)=\perp, \operatorname{Post}_{\tau}^{-}\left(p_{n}\right)=\top\right)$, but if he draws one black ball then there is one black ball less $\left(\operatorname{Post}_{\sigma}^{+}\left(p_{i}\right)=p_{i+1}\right.$, Post $_{\sigma}^{-}\left(p_{i}\right)=\neg p_{i+1}$ for all $\left.i<n\right)$, otherwise the number of black balls remains the same $\left(\operatorname{Post}_{\tau}^{+}\left(p_{i}\right)=p_{i}, \operatorname{Post}_{\tau}^{-}\left(p_{i}\right)=\neg p_{i}\right.$ for all $\left.i<n\right)$.

\subsection{The Update Mechanism}

Definition 4. Given a pd-model $M=\left(W,\left\{\sim_{j} ; j \in G\right\},\left\{P_{j} ; j \in G\right\}, V, w_{0}\right)$ and a generic action model $\boldsymbol{\Sigma}=\left(\Sigma, S,\left\{\sim_{j} ; j \in G\right\},\left\{P_{j}^{\Gamma} ; \Gamma\right.\right.$ is a m. c. subset of $S$ and $j \in G\},\left\{\right.$ Post $\left.\left._{\sigma} ; \sigma \in \Sigma\right\}, \sigma_{0}\right)$, we define their update product to be the pd-model $M \otimes \Sigma=\left(W \otimes \Sigma,\left\{\sim_{j}^{\prime} ; j \in G\right\},\left\{P_{j}^{\prime} ; j \in G\right\}, V^{\prime}, w_{0}^{\prime}\right)$, where:

1. $W \otimes \Sigma=\left\{(w, \sigma) \in W \times \Sigma ; P_{j}^{w}(\sigma)>0\right\}$.

2. $(w, \sigma) \sim_{j}^{\prime}(v, \tau)$ iff $w \sim_{j} v$ and $\sigma \sim_{j} \tau$.

3. We set

$$
P_{j}^{[w]_{j}}(\sigma)=\frac{\sum\left\{P_{j}(v) \cdot P_{j}^{v}(\sigma) ; w \sim_{j} v\right\}}{\sum\left\{P_{j}(v) \cdot P_{j}^{v}(\tau) ; w \sim_{j} v, \sigma \sim_{j} \tau\right\}} .
$$


Then

$$
P_{j}^{\prime}(w, \sigma)=\frac{P_{j}(w) \cdot P_{j}^{[w]_{j}}(\sigma)}{\sum\left\{P_{j}(v) ; w \sim_{j} v \text { and } P_{j}^{v}(\sigma)>0\right\}}
$$

4. $V^{\prime}(p)=\left\{(w, \sigma) \in W \otimes \Sigma ; M, w \models \operatorname{Post}_{\sigma}^{+}(p) \vee\left(p \wedge \neg \operatorname{Post}_{\sigma}^{-}(p)\right)\right\}$.

5. $w_{0}^{\prime}=\left(w_{0}, \sigma_{0}\right)$.

\section{Intuitive Interpretation and Motivations.}

Items 1,2,5 are completely similar to the 'BMS' system ( BMS04 or [Auc05]) except for item 1 where the precondition is replaced by $P_{j}^{w}(\sigma)>0$ (see section above). So, we only motivate items 3 and 4 .

Item 3. We want to determine $P_{j}^{\prime}(w, \sigma)=P_{j}(W \cap A)$ where $W$ stands for 'we were in world $w$ before $\sigma$ occurred' and $A$ for 'action $\sigma$ occurred'. More formally, in the probability space $[(w, \sigma)]_{j}:=\left\{(v, \tau) ;(v, \tau) \sim_{j}(w, \sigma)\right\}, W$ stands for $\left\{(w, \tau) ; \tau \sim_{j} \sigma\right\}$ and $A$ for $\left\{(v, \sigma) ; v \sim_{j} w\right\}$ and we can check that $W \cap A=$ $\{(w, \sigma)\}$.

Probability theory tells us that

$$
P_{j}(W \cap A)=P_{j}(W \mid A) \cdot P_{j}(A) .
$$

We first determine $P_{j}(W \mid A)$, that is to say the probability that $j$ was in world $w$ given the extra assumption that action $\sigma$ occurred in this world. We reasonably claim

$$
P_{j}(W \mid A)=\frac{P_{j}(w)}{\sum\left\{P_{j}(v) ; w \sim_{j} v \text { and } P_{j}^{v}(\sigma)>0\right\}} .
$$

That is to say, we conditionalize the probability of $w$ for $j$ (i.e. $P_{j}(w)$ ) to the worlds where the action $\sigma$ took place and that may correspond for $j$ to the actual world $w$ (i.e. $\left\{v ; w \sim_{j} v\right.$ and $\left.P_{j}^{v}(\sigma)>0\right\}$ ). That is how it would be done in classical probability theory. The intuition behind it is that we now possess the extra piece of information that $\sigma$ occurred in $w$. So the worlds indistinguishable from $w$ where the action $\sigma$ did not occur do not play a role anymore for the determination of the probability of $w$. We can then get rid of them and conditionalize on the remaining relevant worlds.

It remains to determinate $P_{j}(A)$ which we also denote $P_{j}^{[w]_{j}}$; that is to say the probability for $j$ that $\sigma$ occurred. Agent $j$ does not know in which world of $[w]_{j}:=\left\{v ; v \sim_{j} w\right\}$ she dwells. So this action could occur for $j$ in any world $v$ of $[w]_{j}$, each time with probability $P_{j}^{v}(\sigma)$. So we would first be tempted to take the average of them: $P_{j}(A)=P_{j}^{[w]_{j}}(\sigma)=\frac{\sum\left\{P_{j}^{v}(\sigma) ; v \sim_{j} w\right\}}{n}$, where $n$ is the number of world in $[w]_{j}$.

But we have more information than that. $j$ does not know in which world of $[w]_{j}$ she is, but she has a preference among them, which is expressed by $P_{j}$. So we can refine our expression above and take the center of mass (or barycenter) of the $P_{j}^{v}(\sigma) \mathrm{s}$ balanced respectively by the weights $P_{j}(v) \mathrm{s}$ (whose sum equals 1 ), 
instead of taking roughly the average (which is actually also a center of mass but with weights $\left.\frac{1}{n}\right)$. We get $P_{j}(A)=P_{j}^{[w]_{j}}(\sigma)=\sum\left\{P_{j}(v) \cdot P_{j}^{v}(\sigma) ; v \sim_{j} w\right\}$.

Finally, we would naturally want $\sum\left\{P_{j}^{[w]_{j}}(\tau) ; \tau \sim_{j} \sigma\right\}=1$ so that $\left([\sigma]_{j}\right.$, $\mathcal{P}\left([\sigma]_{j}\right), P_{j}^{[w]_{j}}$ ) (where $[\sigma]_{j}:=\left\{\tau ; \tau \sim_{j} \sigma\right\}$ ) forms a (nonstandard) probabilistic space. So we set

$$
P_{j}(A)=P_{j}^{[w]_{j}}(\sigma)=\frac{\sum\left\{P_{j}(v) \cdot P_{j}^{v}(\sigma) ; w \sim_{j} v\right\}}{\sum\left\{P_{j}(v) \cdot P_{j}^{v}(\tau) ; w \sim_{j} v, \sigma \sim_{j} \tau\right\}} .
$$

We then get the expected result by multiplication. We can easily check that its sum on $[(w, \sigma)]_{j}$ is equal to 1 .

Item 4. Intuitively, this formula says that a fact $p$ is true after the performance of $\sigma$ in $w$ iff the condition for $p$ to become true after $\sigma$ was satisfied in $w$, or else if $p$ was already true in $w$ but the condition to switch it to false was not satisfied in $w$.

Remark 2. Note the difference between $P_{j}^{w}(\sigma)$ and $P_{j}^{[w]_{j}}(\sigma)$ : the former is determined by what is true in $w$ and the latter by what $j$ believes in $w$ (see Examples 4 and 5 below). Moreover, since what $j$ believes is the same in every world of $[w]_{j}$, we indeed have $P_{j}^{[w]_{j}}(\sigma)=P_{j}^{\left[w^{\prime}\right]_{j}}(\sigma)$ for all $w^{\prime} \in[w]_{j}$.

From this product mechanism, we can easily define a dynamic language in the line of BMS04 or Auc05 that we do not spell out here.

Example 4. Let us come back to our 'urn' Example 1. Assume now that A draws a (white) ball from the urn, looks at it and puts it in his pocket, action depicted in Fig.3. Then because B does not have any particular preference about the distribution of the urn, she should believe equally that A draws a black ball and a white ball (see introduction). That is indeed the case : $P_{B}^{\left[w_{i}\right]_{B}}(\sigma)=P_{B}^{\left[w_{i}\right]_{B}}(\tau)=$ $\frac{1}{2}$. Note the difference between $P_{B}^{w_{i}}(\sigma)\left(=\frac{i}{n}\right)$ and $P_{B}^{\left[w_{i}\right]_{j}}(\sigma)\left(=\frac{1}{2}\right)$ (see remark 2).

If we perform the full update mechanism, then we get the pd-model of Fig.3 with probabilities $P_{A}(x)=1$ and $P_{B}(x)=\frac{1}{2 n}$ for all worlds $x$. In this model all the worlds are equally probable for $\mathrm{B}$ and there can not be $\mathrm{n}$ black balls in the urn $\left(p_{n}\right)$ since one has been withdrawn.

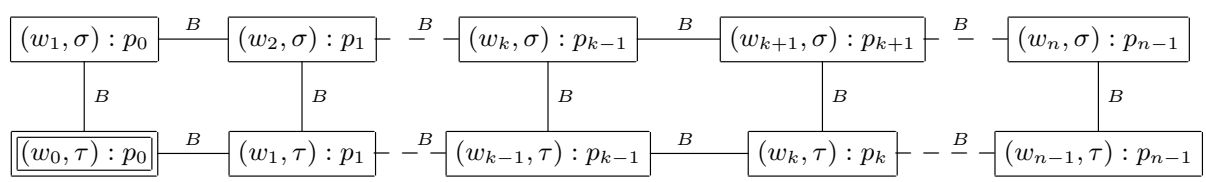

Fig. 3. situation of Examples 1 and 2 after A draws a white ball, looks at it and puts it in his pocket (without specification of the probabilities of worlds for each example: see text). 
Example 5. Let us now come back to our 'urn' Example 2. Assume the same action as above occurs (depicted in Fig.2). However, now B is convinced that there are more black balls than white. Consequently, she should consider it more likely that A draws a black ball than a white ball (see introduction). That is the case indeed: $P_{B}^{\left[w_{i}\right]_{B}}(\sigma)=\frac{3}{4}+\frac{1}{4 k}>P_{B}^{\left[w_{i}\right]_{B}}(\tau)=\frac{1}{4}-\frac{1}{4 k}$.

If we perform the full update, then we still get the pd-model of Fig.3 but with different probability values: $P_{A}(x)=1$ for all worlds $x ; P_{B}\left(w_{i}, \sigma\right)=\varepsilon \cdot\left(\frac{3}{4}+\frac{1}{4 k}\right)$ for $i=1 . . k ; P_{B}\left(w_{i}, \sigma\right)=\frac{1}{k} \cdot\left(\frac{3}{4}+\frac{1}{4 k}\right)$ for $i=k+1 . . n ; P_{B}\left(w_{i}, \tau\right)=\frac{\varepsilon}{4}$ for $i=0 . . k ; P_{B}\left(w_{i}, \tau\right)=\frac{1}{4 k}$ for $i=k+1 . . n-1$. Note the grading of the surprising worlds. It states that $B$ would be a bit more surprised to hear somebody claiming that "there were as many or less black balls than white balls and A drew a white ball" (worlds $\left(w_{i}, \tau\right), i=0 . . k$ ), than to hear somebody claiming that "there were as many or less black balls than white balls and A drew a black ball" (worlds $\left(w_{i}, \sigma\right), i=1 . . k$ ). This is coherent since she believed more that A drew a black ball than she believed that he drew a white ball (i.e. $\left.P_{B}^{\left[w_{i}\right]_{B}}(\sigma)>P_{B}^{\left[w_{i}\right]_{B}}(\tau)\right)$.

\section{Comparisons}

Comparison with the AGM postulates. We can prove that, under the assumption that the AGM notion of 'belief' (see GardRott95]) corresponds to the Lenzen's notion of conviction (see remark 1), the 8 AGM postulates are fulfilled.

Comparison with Reiter's situation calculus. It turns out (surprisingly) that the way we deal with change of facts is completely similar to the way Reiter solves the frame problem in the situation calculus (see Reit01]), and the assumptions he makes about actions are fulfilled in our framework.

Comparison with Kooi's system. Kooi's dynamic probabilistic system (see Kooi03]), based on the static approach by Fagin and Halpern in [FH94, does not make any particular assumption about the relation between probability and epistemic relation (explored in [FH94]); contrary to our approach. Moreover, he deals only with public announcement, and in this particular case our update mechanism is basically the same as his.

Comparison with van Benthem's system. van Benthem's system (see vBen03.) seems similar to ours, but he does not deal with actions changing facts and does not show the influence of beliefs on the interpretation of actions. In that respect, he does not distinguish $P_{j}^{w}(\sigma)$ from $P_{j}^{[w]_{j}}(\sigma)$ as we do (see Remark 2), and his ambiguous $P_{j}^{w}(\sigma)$ seems to be different from ours if we refer to his example. His probabilistic update rule (without motivations) is also different. Besides, his update product can not be iterated because he deals with worlds rather than maximal consistent sets in the probabilities of actions. Anyway, his comparison with the Bayesian setting and other insights are still valid here. 


\section{Conclusion}

We have proposed a system which models belief with probability and whose update performs genuine belief revision (unlike the existing approaches in the literature). So it indirectly offers a new probabilistic approach to belief revision.

Moreover, in our modeling we have introduced surprising worlds necessary to model incorrect beliefs. Their relative degrees of surprise for the agent is expressed by infinitesimals. This use of infinitesimal has also enabled us to express what would surprise the agent and by how much. This is of importance since we want to describe with most accuracy any epistemic state of mind of any agent, including what would surprise her. In that respect the notion of knowledge can also be added to this dynamic epistemic system, which then validates all the Lenzen's axioms for belief and knowledge (that I consider as most accurate and expressive to describe epistemic states).

Finally, we have also incorporated actions that change facts of the situation and showed from a formal point of view how our beliefs can affect our interpretation of actions. In a sense this last point complements and reverses the classical view whereby only our interpretation of actions affects our beliefs and not the other way around, as in belief revision theory.

\section{References}

[Auc05] G. Aucher. A combined System for Update Logic and Belief Revision. In M. W. Barley and N. Kasabov (Eds.): PRIMA 2004, LNAI 3371 Pages 1-17, 2005.

[Adams75] E. W. Adams. The Logic of Conditionals. In Synthese Library Volume 86, D. Reidel, Dordrecht, Netherlands, 1975.

[BMS04] A. Baltag, L.S. Moss,and S. Solecki. Logic for epistemic program. In Synthese Volume 139(2) Pages: 165 - 224.

[FH94] R. Fagin and J. Y. Halpern. Reasoning about knowledge and probability. In Journal of the ACM (JACM) Volume 41(2) Pages: 340 - 367.

[GardRott95] P. Gardenfors and H. Rott, 1995, 'Belief Revision', in D. M. Gabbay, C. J. Hogger and J. A. Robinson, eds., Handbook of Logic in Artificial Intelligence and Logic Programming 4, Oxford University Press, Oxford 1995.

[Keis86] H. J. Keisler. Elementary Calculus: An Approach Using Infinitesimal. Prindle, Weber and Schmidt (eds), 1986. Online edition on the website http://www.math.wisc.edu/ keisler/calc.html .

[Kooi03] B.P. Kooi (2003). Probabilistic Dynamic Epistemic Logic. In Journal of Logic, Language and Information Volume 12(4) Pages: 381-408.

[Len03] W. Lenzen. Knowledge, Belief, and Subjective Probability: Outlines of a Unified System of Epistemic/Doxastic Logic. In: V. F. Hendricks and Al. (eds.), Knowledge Contributors, Dordrecht (Kluwer) 2003, Pages: 17-31.

[Reit01] R. Reiter. Knowledge in Action: Logical Foundations for Specifying and Implementing Dynamical Systems, MIT Press, 2001.

[vBen03] J. van Benthem. Conditional probability meets update logic. In Journal of Logic, Language and Information Volume 12(4) Pages: 409 - 421. 